\title{
PTEN in the Stroma
}

\author{
Katie A. Thies, ${ }^{1}$ Julia E. Lefler, ${ }^{2,3}$ Gustavo Leone, ${ }^{2,3}$ and Michael C. Ostrowski ${ }^{2,3}$ \\ ${ }^{1}$ Department of Radiation Oncology and The Comprehensive Cancer Center, The Ohio State University, \\ Columbus, Ohio 43210, USA \\ ${ }^{2}$ Department of Biochemistry and Molecular Biology, Medical University of South Carolina, Hollings Cancer \\ Center, Charleston, South Carolina 29425, USA \\ ${ }^{3}$ Hollings Cancer Center, Medical University of South Carolina, Charleston, South Carolina 29425, USA \\ Correspondence: ostrowsk@musc.edu
}

\begin{abstract}
Although tremendous progress has been made in understanding the functions of Phosphatase and tensin homolog deleted on chromosome 10 (PTEN) in tumor cells, only recently have tumor cell-non-autonomous PTEN actions within the tumor microenvironment (TME) been appreciated. While it is accepted that the TME actively communicates with cancer cells to influence disease progression, our understanding of the genes and pathways responsible is still evolving. Given that inactivation of PTEN in the stroma is correlated with worse outcomes in human cancers, determining the unique functions and mechanisms of PTEN regulation in various TME cell compartments is essential. In this review, the evidence for PTEN function in different TME cell compartments, the mechanisms governing PTEN inactivation, and the downstream pathways regulated by PTEN that are critical for intracellular communication, are covered. The potential clinical implications of these findings as well as the future directions for the study of stromal PTEN are discussed.
\end{abstract}

$P^{\text {hosphatase and tensin homolog deleted on }}$ Pchromosome $10(P T E N)$ is a well-described tumor suppressor gene. Germline PTEN mutations are observed in patients with PTEN hamartoma tumor syndrome (PHTS), which includes Cowden syndrome, Bannayan-Riley-Ruvalcaba syndrome, and Proteus syndrome (Yehia et al. 2019). Patients with Cowden syndrome are predisposed to malignancy, including breast, thyroid, and skin cancers (Liaw et al. 1997; Tan et al. 2012). PTEN is one of the most frequently disrupted genes in sporadic tumors, with profound impacts on cancer progression. As a lipid and protein phosphatase that primarily deactivates phosphoinositide substrates required for PI3K signaling, PTEN negatively regulates the
AKT/PKB survival pathway. PTEN nuclear activity is also important for tumor suppression. Through its enzymatic activities, PTEN governs a number of cellular process including energy metabolism, proliferation, survival, migration, and genomic stability (Song et al. 2012; Milella et al. 2015; Lee et al. 2018).

Work over the last few decades demonstrates the profound influence of the tumor-associated stroma on cancer progression and metastasis. The stroma or tumor microenvironment (TME) consists of a number of different cell types including inflammatory cells, fibroblasts, and endothelial cells as well as the extracellular matrix (ECM). Bidirectional communication between tumor cells and stromal elements is

Editors: Charis Eng, Joanne Ngeow, and Vuk Stambolic

Additional Perspectives on The PTEN Family available at www.perspectivesinmedicine.org

Copyright (C) 2019 Cold Spring Harbor Laboratory Press; all rights reserved; doi: 10.1101/cshperspect.a036111

Cite this article as Cold Spring Harb Perspect Med 2019;9:a036111 
K.A. Thies et al.

critical to tumor initiation, progression, and metastasis (Hanahan and Coussens 2012). While inactivation of classic tumor suppressors in cancer cells can have significant effects on the recruitment and activation of cells within the surrounding microenvironment, seminal work uncovered the non-cell-autonomous function of TGFBRII, TP53, and PTEN in suppressing tumor growth from within stromal cells (Bhowmick et al. 2004; Ajani and Allgood 2005; Trimboli et al. 2009; Lujambio et al. 2013). Notably, the function of PTEN is not restricted to any one particular cell type or tissue, and alterations in PTEN expression are not limited to tumor cells alone. This review will focus on evidence supporting the critical function of PTEN in distinct TME cell compartments, how PTEN is inactivated in the TME cell types, and downstream pathways and effectors that mediate PTEN-dependent communication between stromal cells and the tumor.

\section{PTEN IN STROMAL CELL COMPARTMENTS}

The hereditary mutations in PTEN that result in cancer are not confined to a single cell type, leading to speculation about the role of PTEN in additional cell compartments including fibroblasts, macrophages, T cells, and endothelial cells. Analysis of PTEN expression in human tumor samples suggest PTEN loss is not restricted to tumor cells, and murine models have been used to experimentally demonstrate the noncell-autonomous effects of PTEN on tumor progression. Key evidence supporting PTEN function in the TME is presented below.

\section{Cancer-Associated Fibroblasts}

Cancer-associated fibroblasts (CAFs) play dual roles in the TME, capable of both suppressing tumor onset and promoting tumor growth and metastasis (Kalluri 2016). Our group and others have implicated PTEN loss in stromal fibroblasts as a frequent event associated with worse outcomes in breast, prostate, pancreatic, and endometrial cancers (Kurose et al. 2002; Trimboli et al. 2009; Daikoku et al. 2011; Ashida et al.
2012; Sizemore et al. 2017, 2018; Pitarresi et al. 2018).

Following the observation that PTEN expression in stromal fibroblast (determined by immunohistochemistry [IHC]) was lost in $\sim 25 \%$ of human breast cancer patients, our research team genetically inactivated Pten in stromal fibroblasts of mouse mammary glands using the fibroblast-specific (Fsp-cre) transgene and conditional Pten ${ }^{\operatorname{lox} P}$ alleles (Trimboli et al. 2009). Fibroblast-specific loss of PTEN accelerated the initiation and progression of $M M T V$ ErbB2-driven mammary epithelial tumors. Stromal deletion of Pten in the mouse mammary gland was associated with a remarkable remodeling of the extracellular matrix, increased angiogenesis, and the recruitment of innate immune cells. Notably, global gene expression analysis of mouse mammary stromal cells identified a Ptenspecific signature represented in the tumor-associated stroma of breast cancer patient samples. These experiments were the first to accurately model the human breast TME both at the histological and molecular level.

Loss of fibroblast-specific PTEN has also been reported in the context of pancreatic cancer. Wartenberg et al. (2016) reported that in $\sim 20 \%$ of human PDAC samples PTEN expression was absent in both the tumor and juxtatumoral stroma. Stromal loss of PTEN was highly correlated with metastatic spread of the tumor in this study. Similarly, our group found that PTEN expression was low in the stroma of $\sim 25 \%$ of PDAC patient samples and that this correlated with a mean overall survival of 8.8 months versus 16.9 months in patients scored with high stromal PTEN. Further, patient-derived CAFs with endogenous PTEN knockdown significantly accelerated the growth of PDAC tumor cells compared to control CAFs in an orthotopic coinjection model (Pitarresi et al. 2018). Thus, the loss of PTEN in the microenvironment may be an important collaborator in disease progression.

Similarly, work from Liang and colleagues used cell-type-specific conditional PTEN ablation to reveal a protective role for stromal PTEN in inhibiting endometrial carcinogenesis. The results suggest a mechanism involving increased 
transforming growth factor $\beta$ (TGF- $\beta$ ) signaling in the stroma when Pten is deleted (Liang et al. 2018). Combined, these findings strongly support a tumor-suppressive role for PTEN within tumor-associated fibroblasts. Understanding how PTEN is regulated in fibroblasts and the mechanisms underlying communication with tumor cells and other TME cell compartments are crucial next steps in an effort to strategically target PTEN and PTEN-mediated pathways in stromal fibroblasts.

\section{Endothelial Cells}

Vascular malformations are common in individuals with germline PTEN mutations (Tan et al. 2007). Indeed, PTEN acts to inhibit vascular sprouting and tube formation in vitro, while deletion of Pten in mouse models has the opposite effect (Huang and Kontos 2002). Directly testing whether PTEN in endothelial cells affects tumor progression, heterozygous mutations of Pten in endothelial cells using the Tie2-Cre transgene resulted in enhanced tumor angiogenesis in melanoma and lung cancer models, while homozygous mutations proved embryonically lethal because of aberrant vascular remodeling and hyperproliferation of endothelial cells (Hamada et al. 2005). Both vessel abundance and size were enhanced with PTEN loss in the endothelial cell compartment. This was associated with an increase in endothelial cell proliferation and exaggerated response to Ang-1 or VEGF-A stimulation in vitro (Hamada et al. 2005). Taken together, these results suggest that individuals with hereditary PTEN mutations are not only at risk for developing cancer but that any emerging tumors may show accelerated growth because of increased angiogenesis.

\section{Macrophages}

Macrophages are a critical component of the tumor immune microenvironment, and can promote angiogenesis, immune suppression, tumor cell migration, and metastasis (Yang et al. 2018). A pair of recent studies demonstrated that extracellular vesicles produced by lung or pancreatic cancer cells silenced PTEN expression in macrophages (Hsu et al. 2018; Wang et al. 2018). Loss of PTEN in the macrophages led to a shift to the tumor-promoting M2 phenotype. The M2 macrophages increased lung tumor cell motility in vitro, and pancreatic tumor metastasis in vivo. The tumor-derived extracellular vesicles contained microRNAs, miR-103a (lung) and miR301a-3p (pancreas), which are both known regulators of PTEN expression. Interestingly, these miRs were detected in the circulation of patients and correlated with an increased M2 macrophage population in lung cancer (Hsu et al. 2018), and increased invasion and local metastasis in pancreatic cancer (Hsu et al. 2018; Wang et al. 2018). These findings also establish one of the central mechanisms of PTEN regulation-microRNA-mediated transcript silencing, which is discussed further below.

\section{T Cells}

In addition to being at increased risk for the development of various cancer types, individuals with PHTS are also at higher risk of autoimmune diseases and lymphoproliferative disorders, suggesting the involvement of PTEN in T-cell function (Chen et al. 2017). Heterozygous $\mathrm{Pten}^{+/-}$mice develop a lethal polyclonal autoimmune disorder characterized by activation-induced increases in proliferation and reduced cell death of T cells (Di Cristofano et al. 1999). Pan-T-cell deletion of Pten using Lck-Cre led to a significant increase in thymic cellularity with $\mathrm{T}$ cells showing an activated phenotype (Suzuki et al. 2001; Liu et al. 2010). These mice developed T-cell lymphomas and autoimmune disorders and succumbed to lymphoma within 4-5 months of age. However, if the mice underwent thymectomy, $\mathrm{T}$ lymphomas were $\mathrm{ab}$ sent but severe autoimmune disease did arise (Liu et al. 2010). These findings suggest that PTEN in T cells may modulate self-tolerance.

Especially of interest are studies implicating PTEN function in maintaining $\mathrm{FoxP}^{+}$regulatory $\mathrm{T}$-cell $\left(\mathrm{T}_{\text {reg }}\right)$ activity. Evading the immune system is one of the "hallmarks" of cancer and the immunosuppressive TME is a major barrier to increasing the effectiveness of immunotherapies. $\mathrm{T}_{\text {regs }}$ are a key immunosuppressive compo- 
K.A. Thies et al.

nent of the TME, and previous studies have demonstrated an important function for PTEN in maintaining homeostasis and function of this T-cell population (Huynh et al. 2015; Shrestha et al. 2015). In mouse models of melanoma, lymphoma, and lung cancer, selective deletion of PTEN in $\mathrm{T}_{\text {regs, }}$, using a FoxP3-Cre transgene, reduced tumor growth (Sharma et al. 2015). $\mathrm{T}_{\text {regs }}$ without PTEN failed to express FoxO3a or PD-1 and were converted into proinflammatory helper cells. As a result, $\mathrm{CD}^{+} \mathrm{T}$ cells were activated and dendritic cells (DCs) shifted to the activated myeloid DC phenotype. Of note, treatment of autochthonous melanoma tumors with the small molecule inhibitor of PTEN, VO-OHpic, in combination with either immunotherapy or chemotherapy resulted in an impressive regression of tumors, while any of the three treatments as a monotherapy had very little effect on tumor growth. Thus, ablation of PTEN specifically in $\mathrm{T}_{\text {regs, }}$ compromised the ability of these cells to maintain an activated, immunosuppressive phenotype. While the status of PTEN in $\mathrm{T}_{\text {regs }}$ present in human patient samples was not addressed, these preclinical studies suggest that selective targeting of PTEN may be a potential therapeutic strategy for increasing the effectiveness of immunotherapy. An important caveat is that previous studies that targeted PTEN in $\mathrm{T}_{\text {regs }}$, using a distinct FoxP-Cre knockin-allele resulted in severe autoimmune disease (Huynh et al. 2015; Shrestha et al. 2015). Sharma et al. (2015) also reported autoimmune disease among older mice in their study, albeit apparently less severe than reported in the other two studies.

\section{REGULATION OF PTEN IN THE STROMA}

While much of the work on PTEN regulation is centered on epithelial tumor cells, advances in understanding how PTEN is regulated in the stroma have been made. While general mechanisms may be similar, the precise details of stromal regulation may differ from those in tumor cells. Given the abundant experimental data supporting that PTEN function is exquisitely concentration dependent, there must be a delicate and complex balance to regulate its expres- sion both in cell-specific and context-dependent manners.

\section{Genetic Mechanisms}

Whether stable genetic alterations occur in the stroma of solid tumors remains controversial (Campbell et al. 2009; Eng et al. 2009). For example, while analysis of laser-capture microdissected breast tumor stroma revealed the presence of loss of heterozygosity ( $\mathrm{LOH})$ at the PTEN locus on chromosome 10q23 and even PTEN point mutations (Kurose et al. 2001, 2002), another group failed to detect gross genetic alterations or mutations in the stroma of breast cancer samples (Qiu et al. 2008). These discrepancies may reflect differences in the experimental approaches used ( $\mathrm{Li}$ et al. 2008; Campbell et al. 2009; Eng et al. 2009).

Nonetheless, studies have continued to appear in the literature in support of stromal genetic mechanisms in solid tumors. For example, as noted above, Wartenberg et al. (2016) reported loss of PTEN expression in stromal fibroblasts in $\sim 20 \%$ in of pancreatic cancer samples analyzed. Either loss of one copy of chromosome $10(40 \%)$ or LOH at the PTEN locus (46\%) was detected in the samples lacking stromal PTEN expression. In this study, the combination of fluorescent in situ hybridization, the hybridization-based multiplex ligation-dependent probe amplification technique (MRC-Holland), and direct exome sequencing were used to confirm alterations of PTEN in stromal cells adjacent to the tumor. The study failed to detect PTEN point mutations or PTEN alterations in stroma distal to the tumor. Thus, PTEN haploinsufficiency is frequently associated with loss of PTEN expression in PDAC stromal cells and contributes to silencing.

\section{Epigenetic Mechanisms}

While PTEN can be silenced by mechanisms involving promoter methylation and changes in chromatin structure, this has not been conclusively demonstrated in cells comprising the TME. For example, two comprehensive studies have documented extensive epigenetic alter- 
ations in stromal fibroblasts in breast and pancreatic cancer, but neither reported changes in PTEN (Hu et al. 2005; Xiao et al. 2016). Further, Wartenberg et al. (2016) failed to find methylation of the PTEN promoter in their study.

Perhaps the most well-documented mechanism of stromal PTEN silencing is by means of noncoding microRNAs. In several cancer types, including pancreatic, liver, and breast cancers, the oncogenic microRNA, miR-21, is dysregulated and, importantly, one of its well-defined targets is PTEN. MicroRNA-21 has been shown to be up-regulated in multiple cell compartments and is an important factor in tumor-stroma cross talk as it can be shuttled intracellularly via exosomes. Thus, dysregulation of miR-21 in one cell compartment can have compounding effects by altering signaling pathways, including PTEN/ $\mathrm{AKT}$, in surrounding cells. For instance, in hepatocellular carcinoma, cancer cells secrete exosomal miR-21 that directly targets PTEN in hepatic stellate cells, which, in turn, produce angiogenic cytokines to promote cancer progression (Zhou et al. 2018). Furthermore, laser-capture microdissected patient PDAC tissues revealed miR-21 overexpression and associated PTEN inactivation in tumor-adjacent stroma in pancreatic cancer compared to normal pancreatic tissue and remote stroma, indicating that $\mathrm{miR}-21$ in concert with PTEN haploinsufficiency can lead to silencing of stromal PTEN (Wartenberg et al. 2016).

Additional microRNAs such as miR-301a$3 p$ and miR-103a target PTEN in stromal macrophages. The exosomal transfer of miR-301a$3 p$ from pancreatic tumor cells to macrophages leads to silencing of PTEN and polarization to an M2-like state, promoting tumor metastasis (Wang et al. 2018). Similarly, extracellular vesicles containing miR-103a are shuttled from lung cancer cells to macrophages where miR-103amediated silencing of PTEN results in M2 polarization that enhances both cancer progression and tumor angiogenesis.

A pseudogene of PTEN, PTENP1, has been identified and shares close homology with PTEN messenger RNA (mRNA) (Fujii et al. 1999; Tay et al. 2011; Johnsson et al. 2013). PTENP1 can act as a decoy or competing endogenous mRNA (termed ceRNA) for microRNAs that recognize and silence PTEN, providing a novel mechanism for regulating PTEN expression (Lee et al. 2018). Although the role of PTENP1 in the stroma is unclear, particularly in human cancers, it merits further investigation.

\section{Protein Stability}

Posttranslational modifications to PTEN including acetylation, phosphorylation, and ubiquitination alter its activity and stability, providing additional mechanisms by which PTEN function may be altered in the tumor stroma (AlvarezGarcia et al. 2019). Phosphorylation of PTEN on specific sites in its carboxy-terminal end can either increase stability, or as is the case for Thr366, decrease stability (Alvarez-Garcia et al. 2019). In particular, ubiquitination of PTEN has emerged as a key mechanism regulating its stability, and several E3 ubiquitin ligases have been implicated in regulating PTEN in tumor cells, including NEDD4-1, WWP2, and XIAP-2 (Alvarez-Garcia et al. 2019). Our group recently identified RNF5 as a novel E3 ubiquitin ligase responsible for proteasome-mediated degradation of PTEN in pancreatic fibroblasts lacking the Hedgehog signaling gene, Smoothened (Smo) (Pitarresi et al. 2018). This work demonstrated that increased GSK3 activity, as a result of the loss of Smo, marked PTEN for ubiquitination through phosphorylation of Thr366. An unbiased proteomic screen for PTEN-interacting proteins identified RNF5 as a potential regulator of PTEN stability. RNF5 is a membrane-associated RING finger E3 ligase that, in epithelial cells, is involved in regulating cell motility (Didier et al. 2003), the misfolded protein response (Morito et al. 2008; Tcherpakov et al. 2009), glutamine transport (Jeon et al. 2015), and innate immune responses (Zhong et al. 2009). In pancreatic fibroblasts, RNF5, but not WPP2, is an E3 ubiquitin ligase responsible for marking PTEN for proteasomal degradation when hedgehog signaling is disrupted (Pitarresi et al. 2018). Whether RNF5 is involved in destabilizing stromal PTEN in other types of cancer, and how PTEN degradation may synergize with other mechanisms of PTEN silencing are important questions for future investigations. 
K.A. Thies et al.

\section{PTEN FUNCTIONS IN STROMAL-TUMOR CROSS TALK}

Critical functions of PTEN within the context of the TME, particularly in CAFs, have started to be elucidated. Fibroblast-specific PTEN regulates an oncogenic secretome that can directly affect tumor cells as well as immune cells and endothelial cells within the TME. PTEN in fibroblasts also has profound effects on the structure of the ECM, which shapes intracellular communication in the TME. Several functions of fibroblast-specific PTEN are discussed below and are shown in Figure 1.

\section{Pten/Ets2/miR320 Axis Regulates an Oncogenic Secretome}

As discussed above, studies from Trimboli et al. (2009) demonstrated that deletion of Pten in mammary stromal fibroblasts collaborated with ErbB2 in tumor cells to accelerate initiation and progression of mammary tumors. Gene expression analysis performed on mouse mammary stromal fibroblasts revealed a number of differentially expressed genes upon fibroblastspecific deletion of Pten, including factors involved in ECM remodeling, wound healing, and chronic inflammation (Trimboli et al. 2009). Among the gene expression changes observed upon loss of Pten in stromal fibroblasts, expression and activation of the transcription factor Ets2 was observed. Conditional ablation of both Pten and Ets 2 in mammary fibroblasts significantly decreased the protumorigenic effects of stromal Pten deletion on ErbB2-driven cancer.

To evaluate the tumor-suppressive activity of PTEN in the mammary stroma, global microRNA expression profiling was performed on
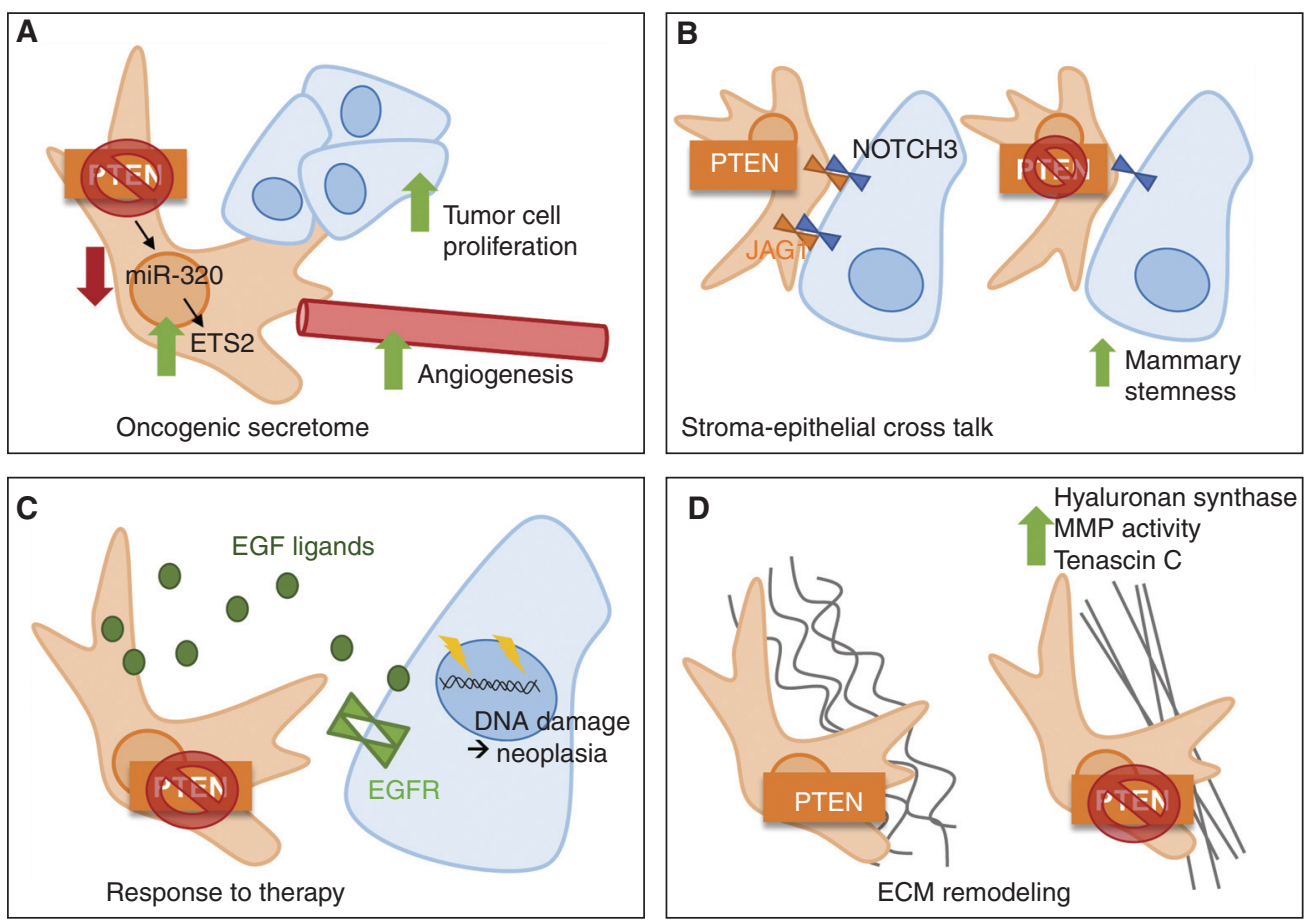

Figure 1. Functions of PTEN in stromal fibroblasts. Stromal PTEN acts to mediate $(A)$ an oncogenic secretome via the PTEN/miR-320/ETS2 axis, $(B)$ cross talk with neighboring epithelium, $(C)$ response to radiotherapy, and $(D)$ extracellular matrix (ECM) remodeling. EGF, Epidermal growth factor; EGFR, EGF receptor. (Panel $A$ is based on data in Bronisz et al. 2012; panel $B$ is based on data in Sizemore et al. 2017; panel $C$ is based on data in Sizemore et al. 2018; and panel $D$ is based on data in Trimboli et al. 2009, Jones et al. 2019, and Pitarresi et al. 2018.) 
wild-type and PTEN-null mouse mammary fibroblasts (MMFs) (Bronisz et al. 2012). One of the microRNA down-regulated in PTEN-null MMFs, miR-320, was also down-regulated in human breast cancer. Interestingly, miR-320 directly targeted ETS2, an essential PTEN target (Bronisz et al. 2012). Importantly, Bronisz and colleagues showed ETS2 is a direct target of miR-320 and that overexpression of miR-320 in PTEN-null fibroblasts overcame protumorigenic effects on tumor cell proliferation in vivo and tumor cell migration in vitro. Using a proteomic approach, a number of secreted factors modulated through the PTEN-miR320-ETS2 axis were identified. Strikingly, this secretome profile could be extrapolated to human breast cancer and used to distinguish normal breast stroma from tumor stroma. Further, the PTEN-miR320-ETS2 secretome profile correlated with recurrence in these breast cancer patients. Taken together, fibroblast-PTEN confers an oncogenic secretome that can influence tumor cell proliferation and migration (Fig. 1A).

Epithelial Cross Talk: PTEN in Fibroblasts Maintains the Stem-Cell Niche and Genomic Stability

Studying epithelial cell populations isolated by fluorescence-activated cell sorting (FACS) from mammary glands with or without fibroblast ablation of PTEN revealed two important pathways that account for collaboration with ErbB2-epithelial cells. First, Pten deletion in fibroblasts led to an expansion of the mammary epithelial stem-cell-enriched population in vivo and increased stem-cell activity as well as abnormal differentiation measured by in vitro assays (Sizemore et al. 2017). Mechanistically, the transmembrane NOTCH signaling ligand, JAGGED-1 was down-regulated in Pten-deleted fibroblasts leading to a reduction in $\mathrm{NOTCH} 3$ receptor signaling within the mammary epithelial compartment and a subsequent increase in the mammary stem-cell-enriched population (Fig. 1B). Three complementary in vitro assays demonstrated increased stem-cell activity, which could be rescued back to control levels by reintroducing JAG1 into Pten-null fibroblasts. Significantly, the pattern of combined low stromal PTEN and low stromal JAG1 is observed in breast cancer patient tissues and correlates with a shorter time to recurrence than for tumor tissues with low levels of either protein alone (Sizemore et al. 2017). These results indicate that PTEN in mammary fibroblasts sustain a mammary stem-cell niche through maintaining paracrine Notch signaling.

Although no changes in luminal cell population numbers were observed with stromal Pten-deletion, gene expression analysis on preneoplastic epithelium from mammary glands revealed DNA repair and mitotic cell-cycle control to be down-regulated in the mature luminal epithelial population from mice lacking fibroblastspecific Pten. Consistent with these results, X-ray radiation of mammary epithelium from stromal Pten-null mice exhibit decreased RAD51 foci formation and persistent $-\mathrm{H} 2 \mathrm{AX}$ foci, suggesting a failure in the DNA damage response (Sizemore et al. 2018). When epithelial cells from these irradiated mice were cultured, an increased incidence of epithelial cells with chromosome breaks and with multicentrosomes were detected from mammary glands with Pten stromal deletion. Mechanistically, Pten-null fibroblasts up-regulate epidermal growth factor (EGF) ligands, which leads to the hyperactivation of EGF receptor (EGFR)/ErbB2 in neighboring epithelium and subsequently induced defective DNA repair in response to irradiation and led to genomic instability (Fig. 1C). The effect of stromal PTEN loss on DNA repair and genomic instability was reduced by pretreatment with the EGFR small molecule inhibitor, erlotinib (Sizemore et al. 2018).

Notably, analysis of a cohort of samples obtained from human breast-reduction procedures revealed that PTEN expression was lost in the stroma of $\sim 25 \%$ of these "normal" human samples. In the Fsp-cre;Pten ${ }^{\text {LoxP/LoxP }}$ mouse model, a single dose of radiation could induce preneoplastic hyperplastic lesions. Remarkably, treatment with erlotinib before radiation exposure prevented the formation of the hyperplastic lesions. Taken together, these findings strongly support a role for stromal PTEN in maintaining 
K.A. Thies et al.

genomic stability in the epithelial compartment of the mammary gland.

Work in pancreatic cancer has similarly demonstrated that RNF5-mediated proteasome degradation of PTEN in pancreatic fibroblasts results in activation of a noncanonical AKT/ GLI2 pathway, which ultimately leads to increased production of the EGF family ligand, TGF- $\alpha$ (Liu et al. 2016; Pitarresi et al. 2018). Consequently, TGF- $\alpha$ activates EGFR signaling in epithelial cells and accelerates pancreatic tumor initiation and progression (Liu et al. 2016; Pitarresi et al. 2018). Whether loss of stromal PTEN by the RNF5-mediated mechanism also results in effects on the stem-cell niche and genomic stability in pancreatic adenocarcinoma remains to be determined.

\section{Pten in Fibroblasts Modulates Extracellular Matrix}

The most striking change in the Fsp-cre; Pten ${ }^{\text {LoxP/LoxP }}$ model was the drastic expansion of a collagen I-rich ECM, even in the absence of the ErbB2 oncogene (Trimboli et al. 2009). Subsequent analysis of changes in the PTENnull fibroblasts secretome revealed that the most abundant secretome components were collagens, matrix metalloproteases, and other proteases, collagen-modifying enzymes, and other ECM components (Fig. 1D). Knockdown of MMP9 or LoxL2 in fibroblasts reduced the ability of the PTEN-regulated secretome to promote migration of epithelial tumor cells in a collagen gel assay (Bronisz et al. 2012). More recent studies have provided additional mechanistic insight into how PTEN influences ECM remodeling. Second harmonic imaging of collagen in both mouse and human tissues demonstrated that loss of PTEN results in thicker collagen fibers and fiber alignment (Jones et al. 2019). In the MMTV-ErbB2; Fsp-cre;Pten ${ }^{\text {LoxP/LoxP }}$ mouse model, collagen fiber orientation was parallel to mammary ducts at preneoplastic stages, but largely perpendicular at the invasive carcinoma stage (Jones et al. 2019). Strikingly, stromal PTEN expression in tissue obtained from breast cancer patients was inversely correlated with higher mammographic density. In vitro assays demonstrated that Pten-null fibroblasts-oriented collagen fibers in parallel streams, and traction force microscopy demonstrated that the PTEN-null fibroblasts exerted increased force on the matrix. Taken together, these findings highlight the importance of PTEN in structuring the ECM to facilitate tumor growth and local invasion.

\section{DISCUSSION AND CONCLUDING REMARKS}

It is evident that PTEN has critical tumor-suppressive functions not only in epithelial cells but also in stromal cells. This leads to the proposition that the PTEN pathway may be an attractive biomarker to identify patients at risk for more aggressive, treatment-resistant disease and potentially as a therapeutic target. Tailoring therapies to target vulnerabilities in both tumor and stromal cells in individual patients may hold promise to decrease the burden of cancer. The PTEN pathway may be an especially attractive target in pancreatic cancer, where the uniquely dense stroma is a barrier to effective therapeutics. A more detailed understanding of the mechanisms that regulate PTEN stability in stromal cells, particularly in fibroblasts, will be required to achieve this goal.

Despite gaps in our knowledge, several potential approaches to restore PTEN function in stromal fibroblasts can be proposed. Targeting E3-ligases such as RNF5 that are responsible for loss of PTEN expression could provide one attractive mechanism for restoring PTEN function. In this regard, homology modeling and virtual ligand screening were successfully used to identify a small molecule inhibitor of RNF5, inh-02 (Sondo et al. 2018). Additional targets regulated by RNF5 in tumor cells have also been implicated in cancer progression, including the unfolded protein stress-responsive pathway and glutamine transport pathways necessary for cancer cell survival and metabolism (Morito et al. 2008; Tcherpakov et al. 2009; Jeon et al. 2015). Ultimately, RNF5 could be an attractive target in both stromal and tumor cells.

Pathways activated downstream of PTEN loss could also provide attractive targets with 
drugs already in the clinic. For example, PTEN loss in stromal fibroblast leads to activation of the PI3K/AKT/mTOR pathway, a pathway with multiple inhibitors already available. Although targeting this pathway in tumor cells has not been successful in the clinic, using them in patient populations with low stromal PTEN could provide additional benefits for tumor-targeted therapies such as radiation or DNA-damaging chemotherapies. Similarly, targeting EGFR signaling with clinically approved inhibitors could improve radiation therapy in patients with loss of stromal PTEN expression (Sizemore et al. 2018). This strategy might be especially relevant for preventing future breast cancer risk in women treated with chest irradiation for childhood cancers (Henderson et al. 2010).

Discovery of a secreted form of PTEN, termed PTEN $\alpha$, may provide a mechanism for restoring PTEN expression in the TME (Hopkins et al. 2013). PTEN $\alpha$ contains an aminoterminal extension of 173 amino acids that arises through alternative translation upstream of the predominant ATG initiation codon. PTEN $\alpha$ can be secreted and taken up by nearby cells. Recently, studies by Russell et al. (2018) demonstrated that incorporation of PTEN $\alpha$ into an oncolytic herpesvirus resulted in restoration of PTEN activity in glioma tumor cells, causing inhibition of PI3K/AKT signaling, priming of the adaptive immune response, and increased regression of orthotopic intracranial tumors. This hybrid herpesvirus could provide a unique reagent to restore PTEN function in the TME.

In conclusion, PTEN expression is frequently lost in the microenvironment in some human cancers and studies in model systems demonstrate that it has critical tumor-suppressive functions from within the TME. Gaps in understanding the precise mechanisms of how PTEN expression is lost remains a major question to be addressed by future studies. In particular, the key questions that remain are what upstream signals trigger PTEN loss, and whether they derive from tumor cells, inflammatory cells, or other sources. Restoring PTEN function or inhibiting select downstream pathways unleashed by PTEN loss in the stroma in combination with tumor-targeting therapies could provide increased benefit to a subset of patients.

\section{ACKNOWLEDGMENTS}

The authors were supported in part by U.S. National Institutes of Health grants to G.L. and M.C.O. (No. P01 CA097189) and Hollings Cancer Center Support Grant No. P30 CA1138313 from the Medical University of South Carolina.

\section{REFERENCES}

Ajani J, Allgood V. 2005. Molecular mechanisms in cancer: What should clinicians know? Semin Oncol 32: 2-4. doi:10.1053/j.seminoncol.2005.07.016

Alvarez-Garcia V, Tawil Y, Wise HM, Leslie NR. 2019. Mechanisms of PTEN loss in cancer: It's all about diversity. Semin Cancer Biol S1044-579X(18)30059-2. doi:10 .1016/j.semcancer.2019.02.001

Ashida S, Orloff MS, Bebek G, Zhang L, Zheng P, Peehl DM, Eng C. 2012. Integrated analysis reveals critical genomic regions in prostate tumor microenvironment associated with clinicopathologic phenotypes. Clin Cancer Res 18: 1578-1587. doi:10.1158/1078-0432.CCR-11-2535

Bhowmick NA, Chytil A, Plieth D, Gorska AE, Dumont N, Shappell S, Washington MK, Neilson EG, Moses HL. 2004 . TGF- $\beta$ signaling in fibroblasts modulates the oncogenic potential of adjacent epithelia. Science 303: 848851. doi:10.1126/science.1090922

Bronisz A, Godlewski J, Wallace JA, Merchant AS, Nowicki MO, Mathsyaraja H, Srinivasan R, Trimboli AJ, Martin CK, Li F, et al. 2012. Reprogramming of the tumour microenvironment by stromal PTEN-regulated miR-320. Nat Cell Biol 14: 159-167. doi:10.1038/ncb2396

Campbell I, Polyak K, Haviv I. 2009. Clonal mutations in the cancer-associated fibroblasts: The case against genetic coevolution. Cancer Res 69: 6765-6768; discussion 6769. doi:10.1158/0008-5472.CAN-08-4253

Chen HH, Händel N, Ngeow J, Muller J, Hühn M, Yang HT, Heindl M, Berbers RM, Hegazy AN, Kionke J, et al. 2017. Immune dysregulation in patients with PTEN hamartoma tumor syndrome: Analysis of FOXP3 regulatory T cells. J Allergy Clin Immunol 139: 607-620.e15. doi:10 .1016/j.jaci.2016.03.059

Daikoku T, Jackson L, Besnard V, Whitsett J, Ellenson LH, Dey SK. 2011. Cell-specific conditional deletion of Pten in the uterus results in differential phenotypes. Gynecol Oncol 122: 424-429. doi:10.1016/j.ygyno.2011.04.022

Di Cristofano A, Kotsi P, Peng YF, Cordon-Cardo C, Elkon KB, Pandolfi PP. 1999. Impaired Fas response and autoimmunity in Pten ${ }^{+-}$mice. Science 285: 2122-2125. doi:10.1126/science.285.5436.2122

Didier C, Broday L, Bhoumik A, Israeli S, Takahashi S, Nakayama K, Thomas SM, Turner CE, Henderson S, Sabe H, et al. 2003. RNF5, a RING finger protein that regulates cell motility by targeting paxillin ubiquitination 
K.A. Thies et al.

and altered localization. Mol Cell Biol 23: 5331-5345. doi:10.1128/MCB.23.15.5331-5345.2003

Eng C, Leone G, Orloff MS, Ostrowski MC. 2009. Genomic alterations in tumor stroma. Cancer Res 69: 6759-6764. doi:10.1158/0008-5472.CAN-09-0985

Fujii GH, Morimoto AM, Berson AE, Bolen JB. 1999. Transcriptional analysis of the PTEN/MMAC1 pseudogene, IPTEN. Oncogene 18: 1765-1769. doi:10.1038/sj.onc .1202492

Hamada K, Sasaki T, Koni PA, Natsui M, Kishimoto H, Sasaki J, Yajima N, Horie Y, Hasegawa G, Naito M, et al. 2005. The PTEN/PI3K pathway governs normal vascular development and tumor angiogenesis. Genes Dev 19: 2054-2065. doi:10.1101/gad.1308805

Hanahan D, Coussens LM. 2012. Accessories to the crime: Functions of cells recruited to the tumor microenvironment. Cancer Cell 21: 309-322. doi:10.1016/j.ccr.2012.02 .022

Henderson TO, Amsterdam A, Bhatia S, Hudson MM, Meadows AT, Neglia JP, Diller LR, Constine LS, Smith RA, Mahoney MC, et al. 2010. Systematic review: Surveillance for breast cancer in women treated with chest radiation for childhood, adolescent, or young adult cancer. Ann Intern Med 152: 444-455; W144-W154. doi:10 .7326/0003-4819-152-7-201004060-00009

Hopkins BD, Fine B, Steinbach N, Dendy M, Rapp Z, Shaw J, Pappas K, Yu JS, Hodakoski C, Mense S, et al. 2013. A secreted PTEN phosphatase that enters cells to alter signaling and survival. Science 341: 399-402. doi:10.1126/ science. 1234907

Hsu YL, Hung JY, Chang WA, Jian SF, Lin YS, Pan YC, Wu CY, Kuo PL. 2018. Hypoxic lung-cancer-derived extracellular vesicle microRNA-103a increases the oncogenic effects of macrophages by targeting PTEN. Mol Ther 26: 568-581. doi:10.1016/j.ymthe.2017.11.016

Hu M, Yao J, Cai L, Bachman KE, van den Brûle F, Velculescu V, Polyak K. 2005. Distinct epigenetic changes in the stromal cells of breast cancers. Nat Genet 37: 899-905 doi:10.1038/ng1596

Huang J, Kontos CD. 2002. PTEN modulates vascular endothelial growth factor-mediated signaling and angiogenic effects. J Biol Chem 277: 10760-10766. doi:10.1074/jbc .M110219200

Huynh A, DuPage M, Priyadharshini B, Sage PT, Quiros J, Borges CM, Townamchai N, Gerriets VA, Rathmell JC, Sharpe AH, et al. 2015. Control of PI(3) kinase in $\mathrm{T}_{\text {reg cells }}$ maintains homeostasis and lineage stability. Nat Immunol 16: 188-196. doi:10.1038/ni.3077

Jeon YJ, Khelifa S, Ratnikov B, Scott DA, Feng Y, Parisi F, Ruller C, Lau E, Kim H, Brill LM, et al. 2015. Regulation of glutamine carrier proteins by RNF5 determines breast cancer response to ER stress-inducing chemotherapies. Cancer Cell 27: 354-369. doi:10.1016/j.ccell.2015.02.006

Johnsson P, Ackley A, Vidarsdottir L, Lui WO, Corcoran M, Grandér D, Morris KV. 2013. A pseudogene long-noncoding-RNA network regulates PTEN transcription and translation in human cells. Nat Struct Mol Biol 20: 440446. doi:10.1038/nsmb. 2516

Jones CE, Hammer AM, Cho Y, Sizemore GM, Cukierman E, Yee LD, Ghadiali SN, Ostrowski MC, Leight JL. 2019. Stromal PTEN regulates extracellular matrix organization in the mammary gland. Neoplasia 21: 132-145. doi:10 $.1016 /$ j.neo.2018.10.010

Kalluri R. 2016. The biology and function of fibroblasts in cancer. Nat Rev Cancer 16: 582-598. doi:10.1038/nrc .2016 .73

Kurose K, Hoshaw-Woodard S, Adeyinka A, Lemeshow S, Watson PH, Eng C. 2001. Genetic model of multi-step breast carcinogenesis involving the epithelium and stroma: Clues to tumour-microenvironment interactions. Hum Mol Genet 10: 1907-1913. doi:10.1093/hmg/10.18 .1907

Kurose K, Gilley K, Matsumoto S, Watson PH, Zhou XP, Eng C. 2002. Frequent somatic mutations in PTEN and TP53 are mutually exclusive in the stroma of breast carcinomas. Nat Genet 32: 355-357. doi:10.1038/ng1013

Lee YR, Chen M, Pandolfi PP. 2018. The functions and regulation of the PTEN tumour suppressor: New modes and prospects. Nat Rev Mol Cell Biol 19: 547-562. doi:10 .1038/s41580-018-0015-0

Li J, Wang L, Mamon H, Kulke MH, Berbeco R, Makrigiorgos GM. 2008. Replacing PCR with COLD-PCR enriches variant DNA sequences and redefines the sensitivity of genetic testing. Nat Med 14: 579-584. doi:10.1038/ nm1708

Liang X, Daikoku T, Terakawa J, Ogawa Y, Joshi AR, Ellenson LH, Sun X, Dey SK. 2018. The uterine epithelial loss of Pten is inefficient to induce endometrial cancer with intact stromal Pten. PLoS Genet 14: e1007630. doi:10 .1371/journal.pgen.1007630

Liaw D, Marsh DJ, Li J, Dahia PL, Wang SI, Zheng Z, Bose S, Call KM, Tsou HC, Peacocke M, et al. 1997. Germline mutations of the PTEN gene in Cowden disease, an inherited breast and thyroid cancer syndrome. Nat Genet 16: 64-67. doi:10.1038/ng0597-64

Liu X, Karnell JL, Yin B, Zhang R, Zhang J, Li P, Choi Y, Maltzman JS, Pear WS, Bassing CH, et al. 2010. Distinct roles for PTEN in prevention of T cell lymphoma and autoimmunity in mice. J Clin Invest 120: 2497-2507. doi:10.1172/JCI42382

Liu X, Pitarresi JR, Cuitiño MC, Kladney RD, Woelke SA, Sizemore GM, Nayak SG, Egriboz O, Schweickert PG, Yu $\mathrm{L}$, et al. 2016. Genetic ablation of smoothened in pancreatic fibroblasts increases acinar-ductal metaplasia. Genes Dev 30: 1943-1955. doi:10.1101/gad.283499.116

Lujambio A, Akkari L, Simon J, Grace D, Tschaharganeh DF, Bolden JE, Zhao Z, Thapar V, Joyce JA, Krizhanovsky V, et al. 2013. Non-cell-autonomous tumor suppression by p53. Cell 153: 449-460. doi:10.1016/j.cell.2013.03.020

Milella M, Falcone I, Conciatori F, Cesta Incani U, Del Curatolo A, Inzerilli N, Nuzzo CM, Vaccaro V, Vari S, Cognetti F, et al. 2015. PTEN: Multiple functions in human malignant tumors. Front Oncol 5: 24. doi:10.3389/fonc .2015 .00024

Morito D, Hirao K, Oda Y, Hosokawa N, Tokunaga F, Cyr DM, Tanaka K, Iwai K, Nagata K. 2008. Gp78 cooperates with RMA1 in endoplasmic reticulum-associated degradation of CFTR $\triangle$ F508. Mol Biol Cell 19: 1328-1336. doi:10.1091/mbc.e07-06-0601

Pitarresi JR, Liu X, Avendano A, Thies KA, Sizemore GM, Hammer AM, Hildreth BE III, Wang DJ, Steck SA, Donohue S, et al. 2018. Disruption of stromal hedgehog signaling initiates RNF5-mediated proteasomal degrada- 
tion of PTEN and accelerates pancreatic tumor growth Life Sci Alliance 1: e201800190. doi:10.26508/lsa .201800190

Qiu W, Hu M, Sridhar A, Opeskin K, Fox S, Shipitsin M, Trivett M, Thompson ER, Ramakrishna M, Gorringe KL et al. 2008. No evidence of clonal somatic genetic alterations in cancer-associated fibroblasts from human breast and ovarian carcinomas. Nat Genet 40: 650-655. doi:10 $.1038 /$ ng. 117

Russell L, Swanner J, Jaime-Ramirez AC, Wang Y, Sprague A, Banasavadi-Siddegowda Y, Yoo JY, Sizemore GM Kladney R, Zhang J, et al. 2018. PTEN expression by an oncolytic herpesvirus directs T-cell mediated tumor clearance. Nat Commun 9: 5006. doi:10.1038/s41467018-07344-1

Sharma MD, Shinde R, McGaha TL, Huang L, Holmgaard RB, Wolchok JD, Mautino MR, Celis E, Sharpe AH, Francisco LM, et al. 2015. The PTEN pathway in $\mathrm{T}_{\text {regs }}$ is a critical driver of the suppressive tumor microenviron ment. Sci Adv 1: e1500845. doi:10.1126/sciadv.1500845

Shrestha S, Yang K, Guy C, Vogel P, Neale G, Chi H. 2015. $T_{\text {reg }}$ cells require the phosphatase PTEN to restrain $T_{H} 1$ and $\mathrm{T}_{\mathrm{FH}}$ cell responses. Nat Immunol 16: 178-187. doi:10 1038/ni.3076

Sizemore GM, Balakrishnan S, Hammer AM, Thies KA, Trimboli AJ, Wallace JA, Sizemore ST, Kladney RD, Woelke SA, Yu L, et al. 2017. Erratum: Stromal PTEN inhibits the expansion of mammary epithelial stem cells through Jagged-1. Oncogene 36: 6658. doi:10.1038/onc .2017 .347

Sizemore GM, Balakrishnan S, Thies KA, Hammer AM Sizemore ST, Trimboli AJ, Cuitino MC, Steck SA, Tozbikian G, Kladney RD, et al. 2018. Stromal PTEN determines mammary epithelial response to radiotherapy. Nat Commun 9: 2783. doi:10.1038/s41467-018-05266-6

Sondo E, Falchi F, Caci E, Ferrera L, Giacomini E, Pesce E, Tomati V, Mandrup Bertozzi S, Goldoni L, Armirotti A, et al. 2018. Pharmacological inhibition of the ubiquitin ligase RNF5 rescues F508del-CFTR in cystic fibrosis airway epithelia. Cell Chem Biol 25: 891-905.e8. doi:10.1016/j .chembiol.2018.04.010

Song MS, Salmena L, Pandolfi PP. 2012. The functions and regulation of the PTEN tumour suppressor. Nat Rev Mol Cell Biol 13: 283-296. doi:10.1038/nrm3330

Suzuki A, Yamaguchi MT, Ohteki T, Sasaki T, Kaisho T, Kimura Y, Yoshida R, Wakeham A, Higuchi T, Fukumoto $\mathrm{M}$, et al. 2001. T cell-specific loss of Pten leads to defects in central and peripheral tolerance. Immunity 14: 523-534. doi:10.1016/S1074-7613(01)00134-0

Tan WH, Baris HN, Burrows PE, Robson CD, Alomari AI, Mulliken JB, Fishman SJ, Irons MB. 2007. The spectrum of vascular anomalies in patients with PTEN mutations: Implications for diagnosis and management. J Med Genet 44: 594-602. doi:10.1136/jmg.2007.048934
Tan MH, Mester JL, Ngeow J, Rybicki LA, Orloff MS, Eng C. 2012. Lifetime cancer risks in individuals with germline PTEN mutations. Clin Cancer Res 18: 400-407. doi:10 .1158/1078-0432.CCR-11-2283

Tay Y, Kats L, Salmena L, Weiss D, Tan SM, Ala U, Karreth F, Poliseno L, Provero P, Di Cunto F, et al. 2011. Codingindependent regulation of the tumor suppressor PTEN by competing endogenous mRNAs. Cell 147: 344-357. doi:10.1016/j.cell.2011.09.029

Tcherpakov M, Delaunay A, Toth J, Kadoya T, Petroski MD, Ronai ZA. 2009. Regulation of endoplasmic reticulumassociated degradation by RNF5-dependent ubiquitination of JNK-associated membrane protein (JAMP). J Biol Chem 284: 12099-12109. doi:10.1074/jbc.M808222200

Trimboli AJ, Cantemir-Stone CZ, Li F, Wallace JA, Merchant A, Creasap N, Thompson JC, Caserta E, Wang H, Chong JL, et al. 2009. Pten in stromal fibroblasts suppresses mammary epithelial tumours. Nature 461: 1084-1091. doi: $10.1038 /$ nature 08486

Wang X, Luo G, Zhang K, Cao J, Huang C, Jiang T, Liu B, Su L, Qiu Z. 2018. Hypoxic tumor-derived exosomal miR301a mediates M2 macrophage polarization via PTEN/ $\mathrm{PI} 3 \mathrm{~K} \gamma$ to promote pancreatic cancer metastasis. Cancer Res 78: 4586-4598. doi:10.1158/0008-5472.CAN-173841

Wartenberg M, Centeno I, Haemmig S, Vassella E, Zlobec I, Galván JA, Neuenschwander M, Schlup C, Gloor B, Lugli A, et al. 2016. PTEN alterations of the stromal cells characterise an aggressive subpopulation of pancreatic cancer with enhanced metastatic potential. Eur J Cancer 65: 8090. doi:10.1016/j.ejca.2016.06.013

Xiao Q, Zhou D, Rucki AA, Williams J, Zhou J, Mo G, Murphy A, Fujiwara K, Kleponis J, Salman B, et al. 2016. Cancer-associated fibroblasts in pancreatic cancer are reprogrammed by tumor-induced alterations in genomic DNA methylation. Cancer Res 76: 5395-5404. doi:10 .1158/0008-5472.CAN-15-3264

Yang M, McKay D, Pollard JW, Lewis CE. 2018. Diverse functions of macrophages in different tumor microenvironments. Cancer Res 78: 5492-5503. doi:10.1158/00085472.CAN-18-1367

Yehia L, Ngeow J, Eng C. 2019. PTEN-opathies: From biological insights to evidence-based precision medicine. $J$ Clin Invest 129: 452-464. doi:10.1172/JCI121277

Zhong B, Zhang L, Lei C, Li Y, Mao AP, Yang Y, Wang YY, Zhang XL, Shu HB. 2009. The ubiquitin ligase RNF5 regulates antiviral responses by mediating degradation of the adaptor protein MITA. Immunity 30: 397-407. doi:10.1016/j.immuni.2009.01.008

Zhou Y, Ren H, Dai B, Li J, Shang L, Huang J, Shi X. 2018. Hepatocellular carcinoma-derived exosomal miRNA-21 contributes to tumor progression by converting hepatocyte stellate cells to cancer-associated fibroblasts. J Exp Clin Cancer Res 37: 324. doi:10.1186/s13046-018-0965-2 


\section{$\&_{\mathrm{CSH}}^{\infty} \&$ Cold Spring Harbor

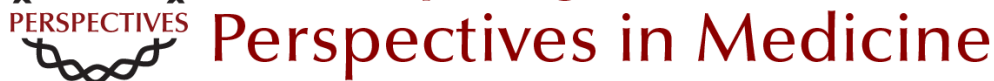

\section{PTEN in the Stroma}

Katie A. Thies, Julia E. Lefler, Gustavo Leone and Michael C. Ostrowski

Cold Spring Harb Perspect Med 2019; doi: 10.1101/cshperspect.a036111 originally published online August 19, 2019

\section{Subject Collection The PTEN Family}

PTEN in Regulating Hematopoiesis and Leukemogenesis

Yilin Wu, Haichuan Zhu and Hong Wu

Connecting Genotype with Behavioral Phenotype

in Mouse Models of Autism Associated with

PTEN Mutations

Amy E. Clipperton-Allen and Damon T. Page

Metabolic Role of PTEN in Insulin Signaling and

Resistance

Yu Zhe Li, Antonio Di Cristofano and Minna Woo

Posttranslational Regulation and Conformational

Plasticity of PTEN

Larissa Kotelevets, Barbara Trifault, Eric Chastre, et al.

Toward Systems Pathology for PTEN Diagnostics Nahal Haddadi, Glena Travis, Najah T. Nassif, et al.

PTEN in Hereditary and Sporadic Cancer Joanne Ngeow and Charis Eng

PTEN Mouse Models of Cancer Initiation and

Progression

Yu-Ru Lee and Pier Paolo Pandolfi

Dual-Specific Protein and Lipid Phosphatase

PTEN and Its Biological Functions

Taojian Tu, Jingyu Chen, Lulu Chen, et al.
PTEN: Bridging Endocytosis and Signaling Matthew F. Lee and Lloyd C. Trotman

PTEN as a Guardian of the Genome: Pathways and Targets

Xinyi Fan, Jeffrey Kraynak, Jonathan P.S. Knisely, et al.

Discovery of the PTEN Tumor Suppressor and Its

Connection to the PI3K and AKT Oncogenes Ramon Parsons

The Complex Landscape of PTEN mRNA

Regulation

Erin Sellars, Martino Gabra and Leonardo Salmena

PTEN Nuclear Functions Jason Ho, Edward S. Cruise, Ryan J.O. Dowling, et al.

Structural Mechanisms of PTEN Regulation Glenn R. Masson and Roger L. Williams

PTEN in Chromatin Remodeling Jingyi Yang and Yuxin Yin

The Role of PTEN in Innate and Adaptive Immunity

Henry Taylor, Arian D. J. Laurence and Holm H. Uhlig

For additional articles in this collection, see http://perspectivesinmedicine.cshlp.org/cgi/collection/ 